tive surgery than those found with MAT with $10 \mathrm{~g}$ load.

Note that the simulation results are in a good agreement with the experimental data from St.-Petersburg Branch IR \& TC «Eye Microsurgery»' and those published in literature, e.g. $[3,4]$.

\title{
References
}

1. Bauer S. M., Karamshina L. A., Kachanov A. B. Mechanical models of the measurements of intraocular pressure by Goldmann and Maklakov applanation tonometers after refractive surgery // Russian Journal of Biomechanics. - 2012. -Vol. 16 (3). - P. 25 31.

2. Iomdina E. N. Mechanical properties of tissues of the human eye // Modern Problems of Biomechanics. Moscow State University. - 2006. - Vol. 11. - P. 183 - 200.

3. Lebedev O. I., Yavorsky A. E. The impact of refractive surgery on the level of intraocular pressure in myopic patients // Russian Journal of Ophthalmology. - 2008. - Vol. 1 (2). - P. $23-25$.

4. Tarutta E. P., Erichev V. P., Larina T. Yu. Controlling intraocular pressure after keratorefractive operations // Proceedings of Ocular Biomechanics - Moscow, 2004. - P. $120-122$.

\section{SIMULATION OF CARDIAC CELL-SEEDED MEMBRANES USING THE EDGE-BASED SMOOTHED FEM}

\author{
Frotscher R. ${ }^{1}$, Gossmann M. ${ }^{2}$, A. Temiz-Artmann ${ }^{2}$, Staat M. ${ }^{1}$ \\ ${ }^{1}$ Aachen University of Applied Sciences \\ Biomechanics Laboratory, Institute of Bioengineering, \\ Heinrich-Mussmann-Strasse 1, 52428 Juelich, Germany \\ frotscher@fh-aachen.de, m.staat@fh-aachen.de \\ ${ }^{2}$ Aachen University of Applied Sciences
}

Laboratory of Cell Biophysics and Laboratory of Medical and Molecular Biology, Institute of Bioengineering, Heinrich-Mussmann-Strasse 1, 52428 Juelich, Germany gossmann@fh-aachen.de, a.artmann@fh-aachen.de

Experimental setup. Since 2001 the Laboratory of Medical and Molecular Biology develops the so-called CellDrum ${ }^{T M}$ device that inflates circular membranes on which cells are cultured and measures its center deflection in order to determine the mechanical effect of the cells on the membrane. As we are interested in the stress of cardiomyocytes we cultivate 2D or 3D cardiac tissue on a circular silicone membrane that is fully clamped in the CellDrum ${ }^{T M}$. After approximately 7 days of cultivation we perform inflation tests producing pressure-deflection curves that enable us to determine not only mechanical properties of the composite tissue and its components but also material parameters for our constitutive model that we apply in simulations.

Kinematics. As the composite tissue is a very thin structure with a radius to thickness ratio between 100 and 2000 it can be modeled as a plate. Using ReissnerMindlin assumptions the 5-parameter model can be described as

$$
\begin{aligned}
& u_{1}\left(x_{1}, x_{2}, x_{3}\right)=u_{1}^{0}\left(x_{1}, x_{2}\right)+x_{3} \theta_{x_{2}}\left(x_{1}, x_{2}\right), \\
& u_{2}\left(x_{1}, x_{2}, x_{3}\right)=u_{2}^{0}\left(x_{1}, x_{2}\right)-x_{3} \theta_{x_{1}}\left(x_{1}, x_{2}\right),
\end{aligned}
$$




$$
u_{3}\left(x_{1}, x_{2}, x_{3}\right)=u_{3}^{0}\left(x_{1}, x_{2}\right) \text {, }
$$

with $u_{1}^{0}, u_{2}^{0}, u_{3}^{0}$ the displacements in the three directions and $\theta_{x_{1}}$ and $\theta_{x_{2}}$ the rotations about the the two in-plane axes respectively as described in [1].

ES-FEM. The geometrically and physically nonlinear simulations are performed using the so-called Edge-based Smoothed Finite Element Method (ESFEM) [1-2]. The principal idea of smoothed FEM is to smooth the strain field over so-called smoothing domains. The smoothed strain field $\bar{\varepsilon}$ on smoothing domain $\Omega_{k}^{s}$ is given as

$$
\bar{\varepsilon}\left(x_{k}\right)=\left\{\begin{array}{l}
\int_{\Omega_{k}^{s}} \tilde{\varepsilon}(x) \mathscr{W}\left(x_{k}-x\right) d \Omega, \text { for } \tilde{\varepsilon}(x) \text { easily available, } \\
\int_{\Omega_{k}^{s}} L_{d} \bar{u}(x) W^{\natural}\left(x_{k}-x\right) d \Omega, \text { else, }
\end{array}\right.
$$

with $\tilde{\varepsilon}$ being the compatible strain field, $L_{d}$ the divergence operator and a scalar weighting function. The discretization is done on the smoothing domains that in the case of ES-FEM are formed by taking two corner nodes of an edge of the finite element mesh and the respective centroids of the neighboring elements. The global tangent stiffness matrix then reads as

$$
\bar{K}_{I}=\sum_{k=1}^{N_{e}}\left(\bar{B}_{k}\right)_{I}^{T} \mathcal{E}\left(\bar{B}_{k}\right)_{J} A_{k}+\sum_{k=1}^{N_{e}}\left(\bar{G}_{k}\right)_{I}^{T} \breve{N}\left(\bar{G}_{k}\right)_{J} A_{k},
$$

with $N_{e}$ the number of edges in the mesh, i.e. the number of smoothing domains, and the nonlinear constitutive tensor $\mathcal{E}$. The stiffness matrix is composed of membrane, bending, shear, 1, membrane-bending coupling and geometric contributions. To avoid shear locking we employ the Discrete Shear Gap method [3]. We implement this method in the open-source industrial finite element software Code_Aster that solves the resulting system of nonlinear equations using the Newton-Raphson algorithm.

The purpose of employing ES-FEM rather than standard FEM is the strong performance of the method when applied to linear triangular elements while keeping an accurate approximation of even complex geometries. Besides that ES-FEM is able to compute problems with highly distorted elements very accurately. As we are dealing with thin soft tissues in most of our applications those properties are essential.

Constitutive model. The silicone membrane as well as the passive part of the cardiac tissue are modeled using an isotropic Neo-Hookean and a volumetric strain energy [4]. The passive strain energy reads as

$$
\Psi_{p}\left(I_{1}^{*}, J\right)=\Psi_{N H}\left(I_{1}^{*}\right)+U(J)=C_{10}\left(I_{1}^{*}-3\right)+k(J-1)^{2} .
$$

In order to capture the active contractile behaviour of the cardiac tissue we employ a simplified version of the model proposed by Hunter et al. [5]. The model assumes an additive split of the second Piola-Kirchhoff stress tensor into a passive part $S_{p}$ that can be derived from equation (1) and an active part 


$$
S_{a}=T\left(t, \lambda,\left[\mathrm{Ca}^{2+}\right]_{i}\right) I,
$$

that depends on time $t$, the current stretch $\lambda$ and the inner Calcium concentration $\left[\mathrm{Ca}^{2+}\right]_{i}$. Due to the circular shape of the membrane there is no preferred fiber direction. Therefor the tissue is assumed to be isotropic. As mentioned earlier the numerous material parameters are determined from inflation experiments, from other measurements or taken from literature. We have implemented this muscle model in Code_Aster.

Results. The smoothed FEM allows us to simulate the inflation experiments in their whole range. Using a least square parameter fitting we can determine passive as well as active material parameters. Applying those parameters in simulations we compute pressure-deflection curves that fit the experiments very well, cf. table 1 . Comparing experimental results, simulations of silicone membranes and simulations of composites we are able to determine the passive and active stress in the cardiac tissue separately.

Table 1 - Deflection of a rectangular plate and the CellDrum computed with different methods

\begin{tabular}{|c|c|c|c|}
\hline \multicolumn{4}{|c|}{ Clamped rectangular plate subjected to a uniform pressure } \\
\hline Analytical & FEM Shell & ES-FEM (Hooke) & ES-FEM (Neo-Hooke) \\
\hline 1,80721 & 1,89995 & 1,80455 & 1,7405 \\
\hline \multicolumn{4}{|c|}{ CellDrum (circular plate) at $150 \mathrm{~Pa}$} \\
\hline Experimantal & $\begin{array}{c}\text { FEM Shell } \\
\text { (Neo-Hooke) }\end{array}$ & ES-FEM (Hooke) & \\
\hline 2,18400 & 2,53153 & 2,33168 & \\
\hline
\end{tabular}

\section{References}

1. Frotscher R., Staat M. Application of an edge-based smoothed finite element method on geometrically non-linear plates of non-linear material // CD-ROM Proceedings of the 6th European Congress on Computational Methods in Applied Sciences and Engineering (ECCOMAS 2012) - Vienna. - 2012.

2. Liu G. R., Nguyen-Thoi T. Smoothed Finite Element Methods. CRC Press, Taylor \& Francis Group. - 2010.

3. Bletzinger K.-U., Bischoff M., Ramm E. A unified approach for shear-locking-free triangular and rectangular shell finite elements // Computers \& Structures. - 2000. - Vol. 75, No. 3. - P. 321 - 334.

4. Holzapfel G. A. Nonlinear Solid Mechanics / A Continuum Approach for Engineering. John Wiley \& Sons Ltd. - 2000.

5. Hunter P. J., McCulloch A. D., ter Keurs H. E. D. J. Modeling the mechanical properties of cardiac muscle // Progress in Biophysics \& Molecular Biology. - 1998. - Vol. 69. - P. $289-331$. 